\title{
Physical Activity is a Medicine for Non-Communicable Diseases: A Survey Study Regarding the Perception of Physical Activity Impact on Health Wellbeing
}

This article was published in the following Dove Press journal:

Risk Management and Healthcare Policy

\section{Zulkaif Ahmed Saqib (iD) \\ Jianhui Dai ${ }^{2}$ \\ Rashid Menhas ${ }^{3}$ \\ Shahid Mahmood ${ }^{4}$ \\ Maria Karim ${ }^{5}$ \\ Xuehui Sang $\mathbb{D}^{2}$ \\ Yu Weng (iD ${ }^{2}$}

'Research Institute of Business Analytics \& Supply Chain Management, College of Management, Shenzhen University,

Shenzhen, Guangdong Province 518060, People's Republic of China; ${ }^{2}$ School of Physical Education and Sports, Soochow University, Suzhou 215006, Jiangsu Province, People's Republic of China; ${ }^{3}$ Research Center of Sports Social Sciences, School of Physical Education and Sports, Soochow University, Suzhou 215006, Jiangsu Province, People's Republic of China; ${ }^{4}$ College of Management, Shenzhen University, Shenzhen, Guangdong Province 518060, People's Republic of China; ${ }^{5}$ International Institute of Islamic Economics (IIIE), International Islamic University (IIU), Islamabad, 44000, Islamic Republic of Pakistan

Correspondence: Jianhui Dai School of Physical Education and Sports, Soochow University, No. 50 Donghuan Road, Suzhou 215006 Jiangsu Province, People's Republic of China

Email sddjh@suda.edu.cn

Rashid Menhas

Research Center of Sports Social

Sciences, School of Physical Education and

Sports, Soochow University, No. 50

Donghuan Road, Suzhou 215006 Jiangsu

Province, People's Republic of China

Email menhas.r@yahoo.com
Background: A modifiable risk factor for many diseases is physical inactivity. In this modern era, physical activity is acknowledged as a vital strategy to promote good health. According to the WHO, regular physical activity has various benefits for psycho-physical health. Decreased physical inactivity is happening in China due to the increased participation of the Chinese population in physical activity. The human body needs a specific level of physical activity to maintain good health.

Objective: The main aim of the present study was to assess the Chinese people's understanding of physical activity and participation in physical activity as a preventive measure against different diseases.

Methods: The present study was exploratory and conducted in the Kunshan county-level city in Jiangsu Province, China. A well-designed questionnaire was used to collect the data from the survey participants. A total of 3390 residents were selected from 1300 households through a simple random sampling technique. The collected data were analyzed through SPSS software. Both univariate and bivariate analysis were performed.

Results: The survey results showed that the majority of women are participating in physical activity. Most of the Kunshan people participate in physical activity one time or more than one time per week. They perform 30-60 minutes of moderate-intensity physical activity to improve physical fitness, recreation, and disease prevention. Fitness walking is the most common type of physical activity among Kunshan people, and most of them participate in physical activity at night. Wilcoxon signed-rank test indicated that post-test ranks are statistically higher than pre-test ranks $(\mathrm{P}<0.0039,0.0039,0.0039,0.0020,0.0001,0.0156$, 0.0313).

Conclusion: The study results showed that physical activity plays a catalyst role in improving human health on a natural path. It reduces the risk factor of non-communicable diseases and promotes general wellbeing. These findings show that physical activity level increases in Chinese society as more people participate in physical activity for health-being. Keywords: physical activity, health, diseases, understanding, participation, wellbeing

\section{Introduction}

Physical activity is an organized activity and is further divided into different categories (transport/work/leisure) according to the purpose. The primarily physical activity is aimed at physical and health capacity improvement. ${ }^{1}$ Physical activity is 
defined as any bodily movement produced by skeletal muscles that require energy expenditure. ${ }^{2}$ Those activities are included in sustainable physical activity, performed with sufficient duration, frequency, and intensity to promote good health and wellbeing. ${ }^{3}$ Further, physical activity is a universal concept and defined as "bodily movement produced by the contraction of skeletal muscles, and that substantially increases the amount of energy you expend"4 According to the World Health Organization, the first indicator of health is physical activity at the community level. ${ }^{5}$ The risk of many chronic diseases such as cancer, cardiovascular condition, and diabetes (type 2) reduce through regular physical activity. ${ }^{6}$ The sedentary lifestyle is the leading cause of the increased mortality rate globally, and WHO stated that about 3.2 million deaths per year are occurring due to physical inactivity across the world. ${ }^{7}$ Physical activity plays a fundamental role in balancing energy and weight control. About $81 \%$ of schoolgoing adolescents and $23 \%$ of adults have insufficient physical activity across the globe. In general, girls and women are less physically active than boys and men, and older adults are also inadequate physical activity than younger adults. ${ }^{8}$ In developing and developed countries, chronic health conditions and non-communicable diseases are linked with physical inactivity. ${ }^{9}$ Blair et al found that physical inactivity, chronic health conditions, and low cardiovascular fitness have a direct link. ${ }^{10}$ High blood pressure, high blood glucose, smoking, obesity, and physical inactivity are the five main leading factors of death, as indicated in Figure 1. ${ }^{1}$

According to the WHO, regular physical activity has various benefits for psycho-physical health. At all stages of life, physical activity plays an essential role in the prevention of non-communicable diseases. Physical activity has a strong relationship with major non-communicable diseases. The member countries of WHO are agreed to reduce relatively about $10 \%$ of low physical activity prevalence by 2025 . It is one of the essential global targets for

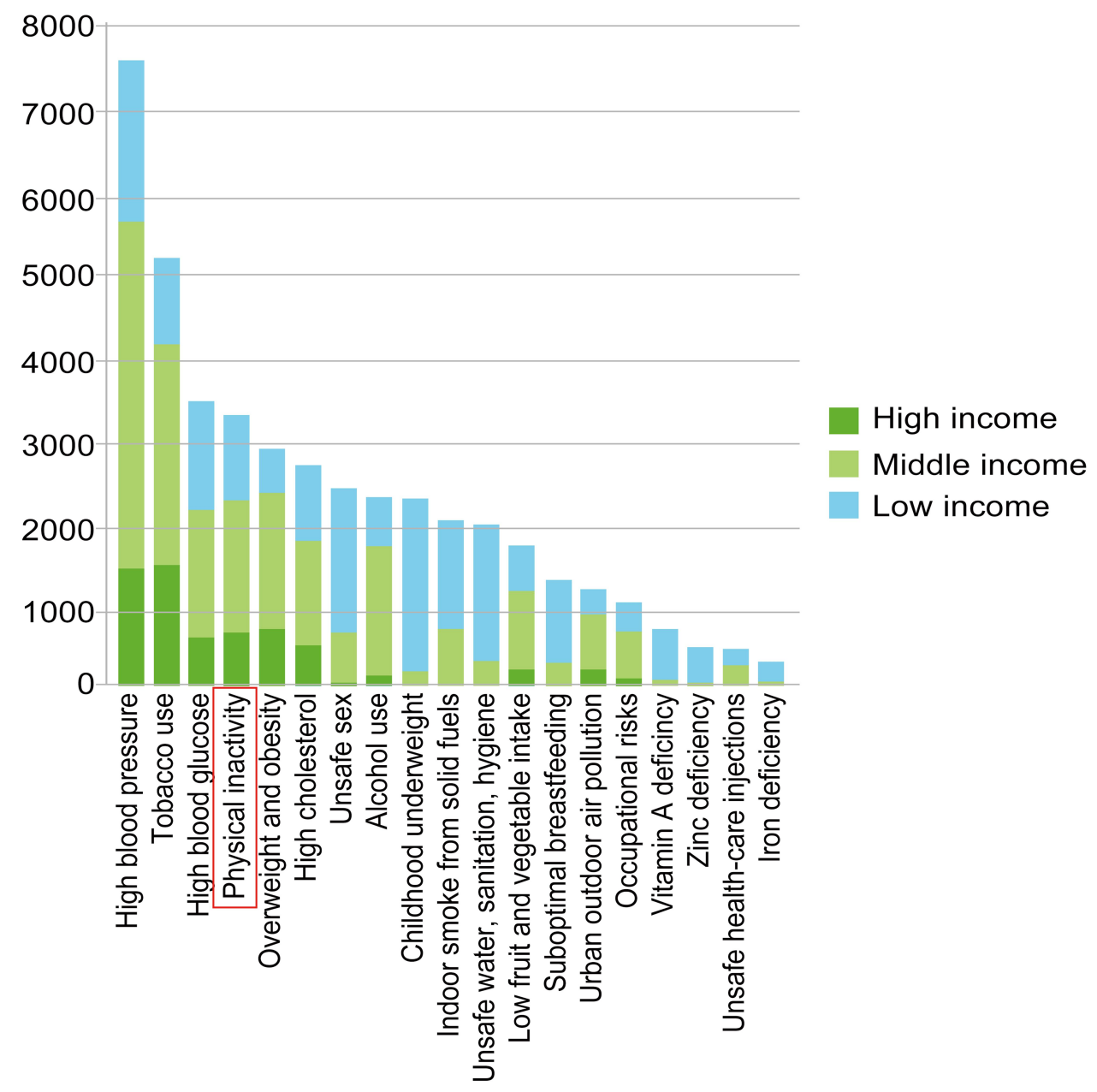

Figure I Non-communicable diseases. 
improving the preventive, diagnostic, and treatment measures of non-communicable diseases. ${ }^{11}$ Globally, physical inactivity is the main reason behind the obesity epidemic, which is further resulting in spreading chronic diseases across the world.

To promote active and healthier societies, WHO launched a program in June 2018 with the title of "More active people for a healthier world." This program's primary plan is to create more active and healthier societies by improving spaces and places for physical activity. The improved spaces and areas for physical activity will provide more opportunities for every age group to do dance, sport, walking, cycling, and play. ${ }^{12}$ Physical inactivity has become a challenge due to the rising rate of non-communicable diseases. WHO has launched a global action plan to decrease physical inactivity and sedentary behavior in 2025 , about $10 \%$, and in 2030 , about $15 \%$. Physical activity has become part and parcel of a healthy life and living. An adult needs to do moderate-intensity sustainable physical activity at least 150 minutes throughout the week, according to the WHO recommendations. ${ }^{13}$

In this modern era, physical activity has become the priority in public health from childhood to adults, from a young age to an older age among men and women. ${ }^{14}$ Globally people are less active due to technological advancement, social changes, and economic growth. In ancient times people were more active because they need to find food (hunting and gathering) and fight or flight for safety. In this technologically advanced era, studies explored that leisure activity and sports levels have increased. Physical activity is allied with transportation, housework, or any other human body act that needs energy for consumption due to different economic growth interventions for economic growth. ${ }^{15}$ Modern technological advancement is the main factor in declining the level of physical activity in developed countries like the USA and developing countries, for example, China. Physical activity level was decreased by about $35 \%$ in men and $46 \%$ in women from 1991 to 2006 in China. ${ }^{16}$

Globally, physical inactivity is the main reason behind the obesity epidemic, which is further resulting in spreading chronic diseases across the world. The prevalence of physical inactivity was from 2001 to 2016 in Western highincome countries by about 5\% (from 30.9\%, 26.4-38.1 in 2001, to $36 \cdot 8 \%, 34 \cdot 6-38 \cdot 4$, in 2016). Furthermore, in East and Southeast Asia also decreased high-income 5\% (from $25 \cdot 7 \%, 20 \cdot 6-29 \cdot 4$, in 2001 , to $17 \cdot 3 \%, 15 \cdot 8-22 \cdot 1$, in 2016 ). The reduced physical inactivity is happening in the East and
Southeast region due to increased participation in the Chinese population's physical activity and the increasing use of parks among the rapidly growing Chinese elderly population. ${ }^{17}$ According to Professor Morris's demonstration in the 1950s, the men who performed different work requiring a specific level of physical activity suffered less coronary heart disease than those who did sedentary kind of jobs. ${ }^{18}$ Many studies that have been conducted in different countries and populations to explore the benefits of physical activity demonstrated that physical activity is much more useful than medication in some health conditions. ${ }^{19}$ Physical activity and health status have a linear relationship as physical activity increase, then physical fitness and health also improved. ${ }^{20}$ Regular physical activity has a substantial impact on heart health, such as improving blood circulation, improved the contract's contract, and the heart's relaxed ability, which further affects blood pumping effectiveness. Physical activity is also helpful in altering the blood lipid profile, which reduces the risk factor of stroke and heart diseases. ${ }^{21,22}$ Numerous health benefits of physical activity are shown in Figure 2.

The liver and skeletal muscles' functions have a direct relationship with physical activity because it improves insulin sensitivity, resulting in the reduction of type- 2 diabetes. Blood glucose can be better through physical activity in the long and short-term insulin action improvements. ${ }^{23,24}$ Regular physical activity plays a vital role in producing thicker, dense bones, healthy joints, strong muscles, ligaments, and tendons. Physical activity is significant in childhood and adolescence because of physical and bodily growth, which further ensures the proper development of musculoskeletal health during adulthood and old age. Moreover, physical activity reduces the risk of falls and hip fractures in old age and keeps active, further strengthening the body's functional strength, such as climbing stairs, lifting, and carrying. ${ }^{25}$ Moreover, after observing the role of physical activity in improving health and preventing or reducing the risk factors of different diseases, the present study was designed.

\section{The Rationale of the Study}

Physical activity is a catalyst for good health and wellbeing. To maintain good health, human biology needs a specific level of physical activity. The less physical inactivity adaptation to human biology would take a long period for biological evolution. The requirements for physical activity are the same as 400,000 years ago for the people living today. ${ }^{26}$ According to the WHO, regular 


\section{PHYSICAL ACTIVITY: SOME OF THE POTENTIAL BENEFITS}

$30 \%$ lower all-cause mortality comparing most active individuals with least active. Even 10 minutes of brisk walking a day is likely to reduce mortality by up to $15 \%$, irrespective of baseline fittness

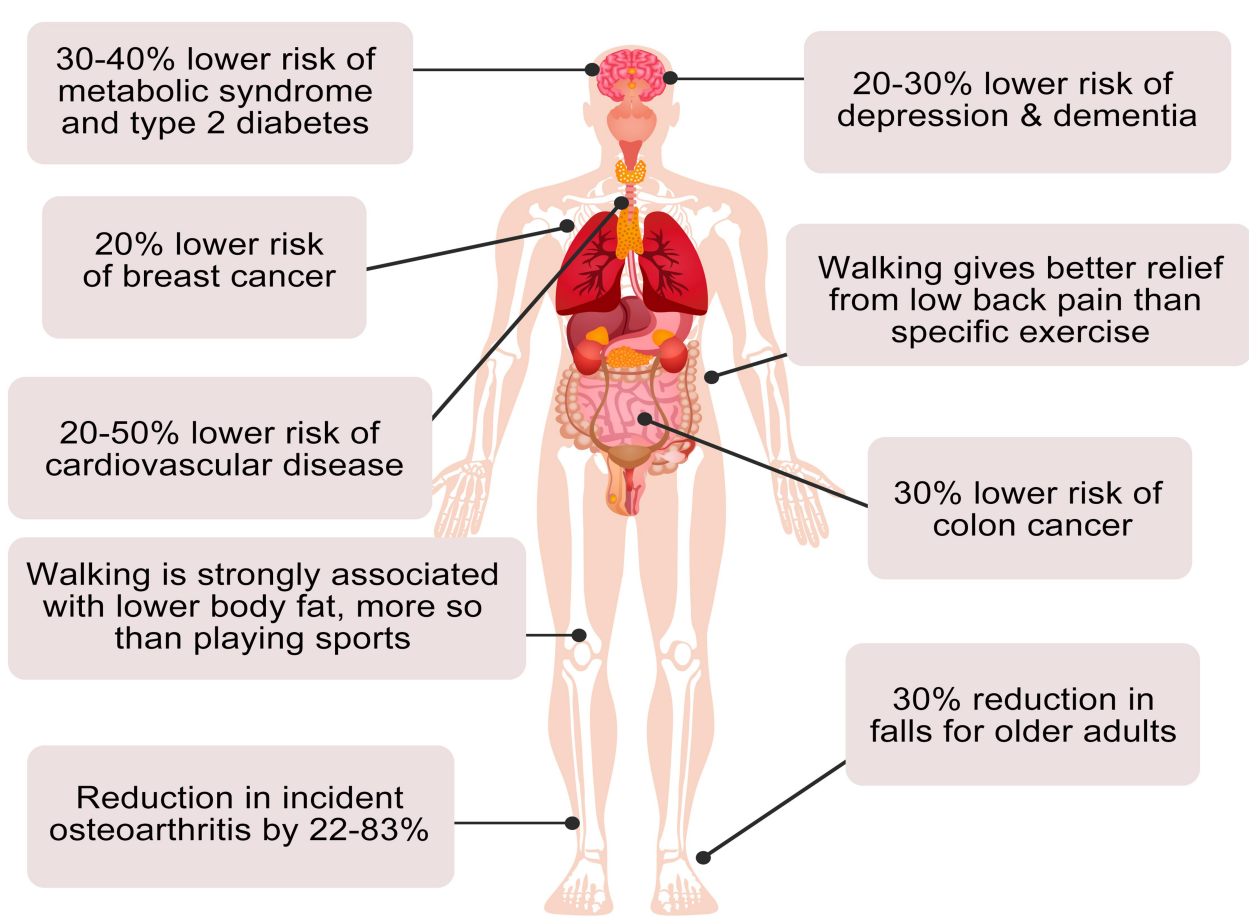

Figure 2 Health benefits of physical activity.

physical activity has various benefits for psycho-physical health. At all stages of life, physical activity plays a vital role in the prevention of non-communicable diseases. Any human body movement, which is resulted in energy consumption or burning calories, improves your health. Physical activity is beneficial for the kind of age group people and helps mental health, improve self-confidence, improve sleep, increase energy, and reduce the risk of chronic diseases. ${ }^{27}$ Regular physical activity helps prevent diseases such as reducing the risk of stroke, heart disease, cancers, high blood pressure, and osteoporosis.

Moreover, physical activity helps ease stress and improves general wellbeing by boosting energy levels. Physical inactivity has become a challenge due to the rising rate of non-communicable diseases. In this context, a global action plan has been launched by WHO to decrease physical inactivity and sedentary behavior in 2025 , about $10 \%$, and in 2030, about 15\%. Physical activity has become part and parcel of a healthy life and living. According to the WHO recommendations, children and young people aged 5-17 years old at least 60 minutes of moderate to vigorous-intensity physical activity daily.
Adults (aged 18-64 years old) need to do moderate-intensity physical activity at least 150 minutes throughout the week. The present study's main objective was to assess the Chinese people's understanding of physical activity and participation in physical activity as a preventive measure against different diseases.

\section{Materials and Methods}

In this section, details of the methodology are presented, which was used to achieve the present study's objectives. The present study's research area was the Kunshan countylevel city under Suzhou city jurisdiction located in the southeast of Jiangsu province, the People's Republic of China, as indicated in Figure 3. The county-level city of Kunshan is a developing, modern metropolis with more than 2000 years of history.

\section{Sampling and Targeted Population}

At the first stage, 1300 households, as shown in Figure 4 of the Kunshan county-level city under Suzhou city's jurisdiction, were selected through a multistage sampling technique. In the second stage, 15 administrative units 


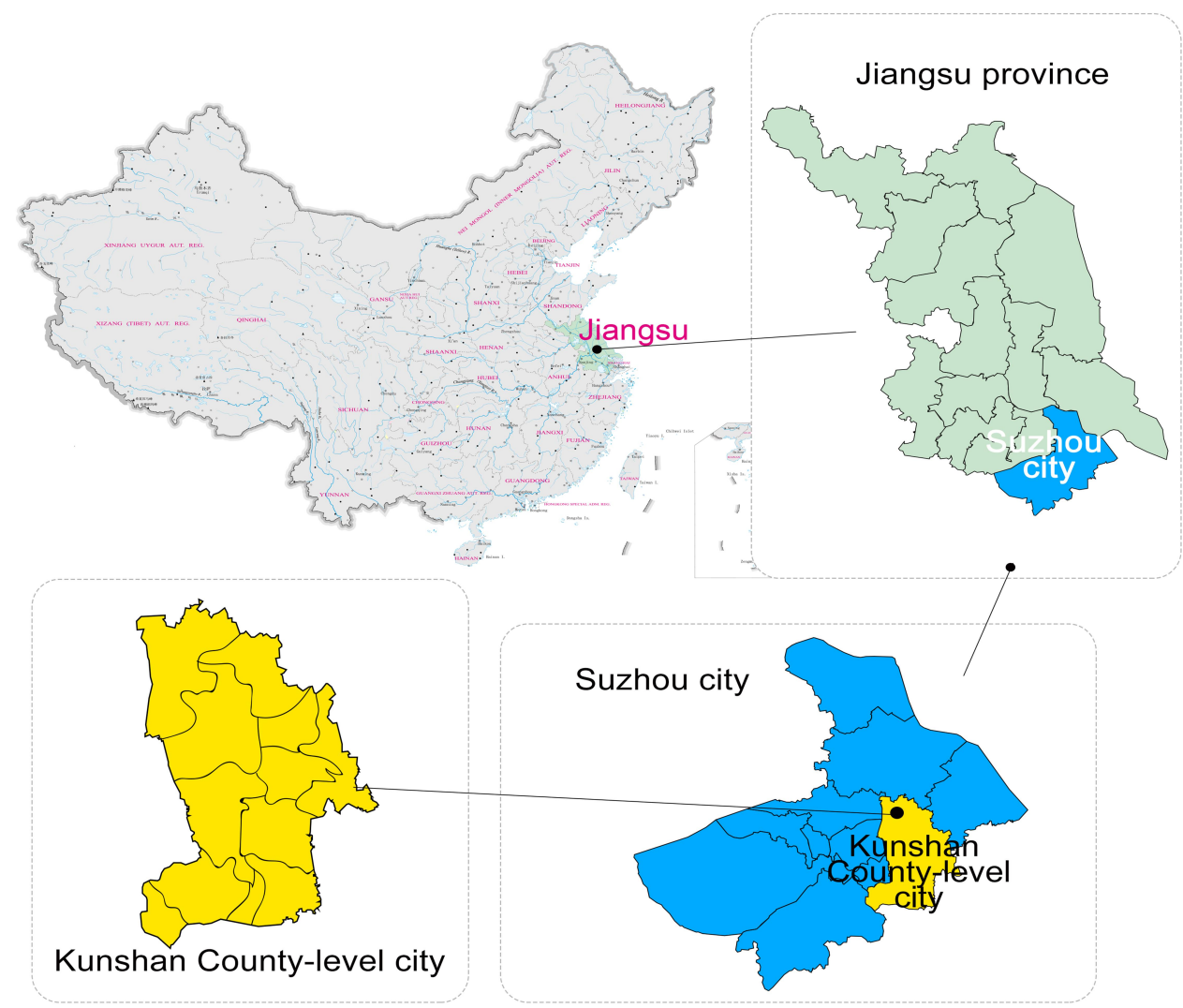

Figure 3 Study locale.
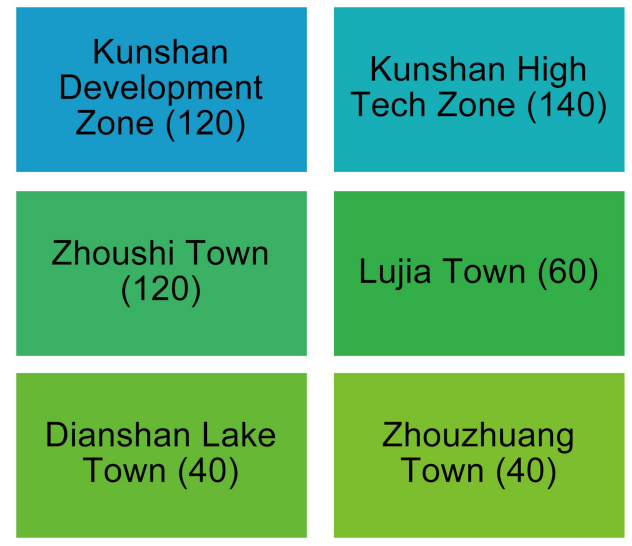

Ting lin (85)
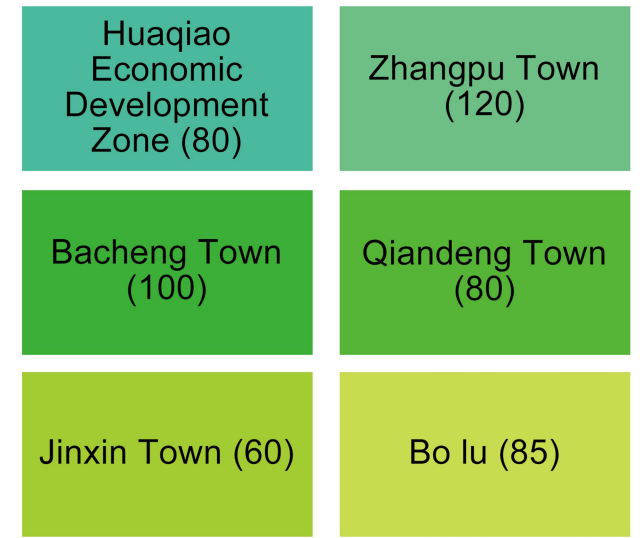

Bo lu (85)

Figure 4 Household distribution.

included towns that have been chosen through simple random sampling techniques. In the third stage, 1300 households from the target population have been selected from, as mentioned in Figure 4, according to the population proportion in each town. At the fourth and final stage,
3390 residents aged 16-70+ were chosen from the 1300 households through a simple random sampling technique. A total of $3171(93.5 \%)$ residents of the Kunshan district participated in the survey, and 219 residents did not participate in the study because of residency ineligibility. The 
survey was conducted among the Kunshan residence population and more than one year of residency was the eligibility to participate in the survey. Written consent had been taken from all survey participants.

\section{Data Collection Tool}

A survey method was used to collect the primary data from the target population. A questionnaire survey is a comprehensive tool for gathering information by asking several questions from a sample of respondents. ${ }^{28} \mathrm{~A}$ welldesigned questionnaire was used as a data collection tool from the survey participants. A group of trained researchers administered face to face interview by using a questionnaire. The questionnaire was based on both openended and closed-ended questions. The pre-testing of the questionnaire was conducted in the study area to check the questionnaire's response level and validity. After pre-testing, some questions were modified for the achievement of better study results. The questions were divided into different segments according to the objective of the study. The question segments were related to demographic information, participation in the physical activity, purpose of the physical activity, physical activity types, the intensity of the physical activity, and perception about physical activity as medicine for NCDs.

\section{Statistical Analysis}

Statistical analysis refers to the method of natural selection, modeling, and transformation, highlighting the useful knowledge and conclusion and supporting the decisionmaking process per the study's goal and objectives. ${ }^{29}$ In the present study, collected data were analyzed through the Statistical Package for Social Sciences (SPSS) version 23.0 (SPSS Inc., Chicago, IL). Both univariate and bivariate analysis were performed. Under the univariate analysis, frequency distribution, percentage, and graphs, techniques were used to summarize the survey data. The Wilcoxon signed-rank test was conducted under the univariate analysis.

\section{Wilcoxon Signed-Rank Test}

The level test signed by Wilcoxon is a non-parametric statistical distributional hypothesis test that checks whether the average grades of samples or the measurements of the specimen differ. ${ }^{30}$ Initially, this test converts the numerical differential values to a signed ranking. Those rankings are based on the differential significance severity. ${ }^{31}$ The statistical assumption is then reached whether or not the mean ranks are equal to zero. ${ }^{32}$
The null and alternative hypothesis for the Wilcoxon rank test can be stated as follows.

$\mathrm{H}_{0}$ : The median difference of the paired is equal to zero.

$\mathrm{H}_{1}$ : The median difference of the paired is not equal to zero.

Estimate each pair difference

$$
D i=X 1 i-X 2 i
$$

Compute the test statistic W

$$
W=\sum_{i=1}^{N r}[\operatorname{sgn}(x 2 i-x 1 i) \cdot R i]
$$

where Ri refers to the ranks.

When the study data are composed of definite scores, the Wilcoxon signed-rank test is used. The Wilcoxon signed-rank test used in the current study to assess a single sample population means ranks differ because data are composed of definite scores. The null and alternative hypothesis for the Wilcoxon rank test of the study is given below.

$\mathrm{H}_{\mathrm{o}}=$ The medians of the population are equal.

$\mathrm{H}_{1}=$ The medians of the population are not equal.

\section{Results}

\section{Univariate Analysis \\ Socioeconomic Characteristics of the Survey Participants}

Table 1 shows that the total survey of 3171 residents in Kunshan city, of which women 1711, accounting for (54.0\%), male 1460, accounting for (46.0\%), the number of female residents participated in the survey slightly higher than men. And Table 1 shows that "30-39 years of age," the largest number of residents, accounting for (26.3\%), followed by "50-59 years old" accounting for (22.4\%), the number of residents " $40-49$ years old" and accounted for (16.2\%), "16-19 years old" and a minimum number of residents, accounting for only (1.3\%). In an educational context, most educated "college (including junior college)," 1268, accounting for (40.0\%), followed by "junior high school education," 757 , accounting for (23.9\%), the minimum number of "graduate students and more than" educated residents, accounting for (1.6\%). Further, Table 1 shows that "other" is the largest number of residents in occupation, there are 874 people, accounting for $(27.5 \%)$, followed by "no occupation (retired, housework, unemployed)" residents, there are 836 people, accounting for $(26.3 \%)$, "the military" at least, only $(0.5 \%)$. 
Table I Socioeconomic Characteristic of the Survey Participants $(N=3 \mid 7 I)$

\begin{tabular}{|c|c|c|c|}
\hline Variables & Category & $\mathbf{N}$ & Percentage \\
\hline \multirow[t]{2}{*}{ Gender } & Male & 1460 & $46.0 \%$ \\
\hline & Female & 1711 & $54.0 \%$ \\
\hline \multirow[t]{7}{*}{ Age } & $16-19$ & 41 & $1.3 \%$ \\
\hline & $20-29$ & 409 & $12.9 \%$ \\
\hline & 30-39 & 833 & $26.3 \%$ \\
\hline & $40-49$ & 515 & $16.2 \%$ \\
\hline & $50-59$ & 711 & $22.4 \%$ \\
\hline & $60-70$ & 477 & $15.0 \%$ \\
\hline & $70+$ & 185 & $5.8 \%$ \\
\hline \multirow[t]{6}{*}{ Education } & Graduate and above & 51 & $1.6 \%$ \\
\hline & University (including junior college) & 1268 & $40.0 \%$ \\
\hline & High school (including college) & 655 & $20.7 \%$ \\
\hline & Junior high school & 757 & $23.9 \%$ \\
\hline & Primary school & 349 & $11.0 \%$ \\
\hline & Illiteracy or low levels of literacy & 91 & $2.9 \%$ \\
\hline \multirow[t]{9}{*}{ Occupation } & State organs, party groups, enterprises and institutions responsible person & 240 & $7.6 \%$ \\
\hline & Professional skill worker & 260 & $8.2 \%$ \\
\hline & Staff and associated personnel & 554 & $17.5 \%$ \\
\hline & Service person & 199 & $6.3 \%$ \\
\hline & Agriculture, forestry, animal husbandry, fisheries and water conservancy production personnel & 59 & $1.9 \%$ \\
\hline & Production, transport equipment operators and related workers & 134 & $4.2 \%$ \\
\hline & Soldier & 15 & $0.5 \%$ \\
\hline & No occupation (retired, housework, unemployed) & 836 & $26.3 \%$ \\
\hline & Other & 874 & $27.5 \%$ \\
\hline
\end{tabular}

\section{Participation in the Physical Activity}

Table 2 shows that most survey participants $(62.7 \%)$ participated in physical activity, while about (37.3\%) did not participate in any physical activity. And almost (50.6\%) participated only once or more per week. In comparison, about (49.4\%) participated less than once a week or did not participate in physical activity. In many countries, the recommended level of physical activity for men and women, adults and children are the same. In this context, 30 minutes for adults per day and 60 minutes moderate-vigorous physical activity per day. Table 2 shows that the majority of the survey participants about (49.79\%) performed physical activity 30 to 60 minutes, about (34.44\%) achieved 60 minutes and more, and only about (15.77\%) performed physical activity less than

Table 2 Participation in the Physical Activity $(\mathrm{N}=3$ I7I)

\begin{tabular}{|l|l|l|l|}
\hline Variables & Category & N & Percentage \\
\hline Participate in the Physical Activity & Yes & 1987 & $62.7 \%$ \\
& No & 1184 & $37.3 \%$ \\
\hline Frequency of physical exercise & Less than once a week and do not participate in physical exercise & 1568 & $49.4 \%$ \\
& One time or more per week & 1603 & $50.6 \%$ \\
\hline Duration of Physical Activity & Less than 30 minutes & 500 & $15.77 \%$ \\
& 30 to 60 minutes & 1579 & $49.79 \%$ \\
& 60 minutes and more & 1092 & $34.44 \%$ \\
\hline The intensity of Physical Activity & Low intensity & 946 & $29.83 \%$ \\
& Moderate intensity & 1743 & $54.97 \%$ \\
\hline
\end{tabular}


30 minutes. In the context of physical activity context, the majority of the respondents, about (54.97\%) performed moderate-intensity physical activity, while about (29.83\%) performed low-intensity physical activity, and only about (15.20\%) committed high-intensity physical activity.

\section{Purpose of Participation in the Physical Activity}

Physical activity has multidimensional impacts on health, such as socio-psychological, cognitive, and prevents from noncommunicable diseases. Mental acuity and depression can also be reduced through regular physical activity. Figure 5 shows that most survey participants $(26.43 \%)$ participate in physical activity to improve physical fitness, and about (21.51\%) participate to avoid different diseases. In comparison (20.91\%), participate in increasing physical activity, and about (8.29\%) participate in the physical activity to increase the beauty, weight loss, body sculpting. About (7.85\%) participate in the physical activity to decompress and relax; about (4.98\%) participate in the physical activity to improve motor skills. About (4.26\%) participate in physical activity to become healthy and handsome, about (3.15\%) survey participate in physical activity for social/other. Only about (2.62\%) of participants join in the physical activity for recreation.

\section{Types of Physical Activity Performed by the Survey Participant}

Figure 6 shows the types of physical activity performed by the survey participants. Majority of the participants about
(38.22\%) participate in the fitness walking, about $16.24 \%$ participate in running, about $(6.24 \%)$ participate in table tennis, badminton, tennis and other ball sports, about (4.79\%) participate in square dance, ballroom dancing, dance, folk dance, line dancing, Yangko (traditional Chinese exercises), about (4.35\%) participate in the football, basketball, volleyball and other large ball sports, about (3.34\%) participate in the rope skipping, shuttlecock kicking, about (3.31\%) participate in the riding, about (3.28\%) participate in the swimming, about (2.24\%) participate in the broadcast gymnastics, rhythmic gymnastics, aerobics and athletic gymnastics, about (3.06\%) participate in the Qigong Yi Jin Jing, Ba Duan Jin, Wu Qin Xi, Liu Zi Jue, yoga, bowling, softball, softball throw slow, shuttlecock ball games, about (2.49\%) participate in the strength training, unarmed, equipment, about (2.11\%) participate in the Wushu, Taichi sword, Mulanfun, about (2.81\%) participate in the other, about $(1.64 \%)$ participate in the activity on the national fitness path mountaineering, rock climbing, mountain cross-country, about $(0.82 \%)$ participate in the fencing, dragon boat, dragon and lion dances, and about $(0.35 \%)$ participate in the fighting taekwondo, karate, boxing, judo, wrestling, and sanda. Traditional Chinese sports are well acknowledged as exercise and consider contributing to the prevention and treatment of chronic diseases. Traditional Chinese sports are a form of multidimensional exercise that will be popular worldwide in the health paradigm. Therapeutic exercise is beneficial for health, and traditional Chinese exercise practices are gaining

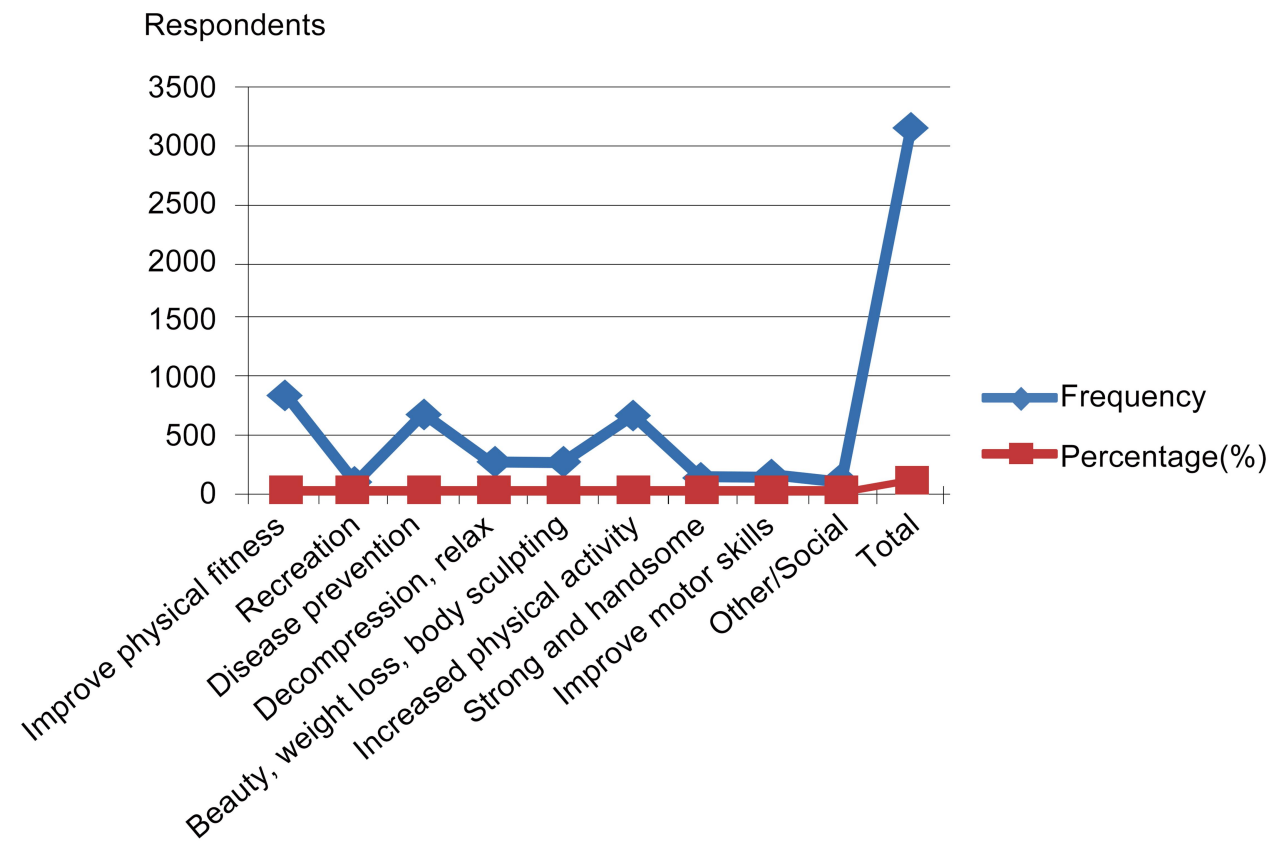

Figure 5 Purpose of participation in physical activity. 


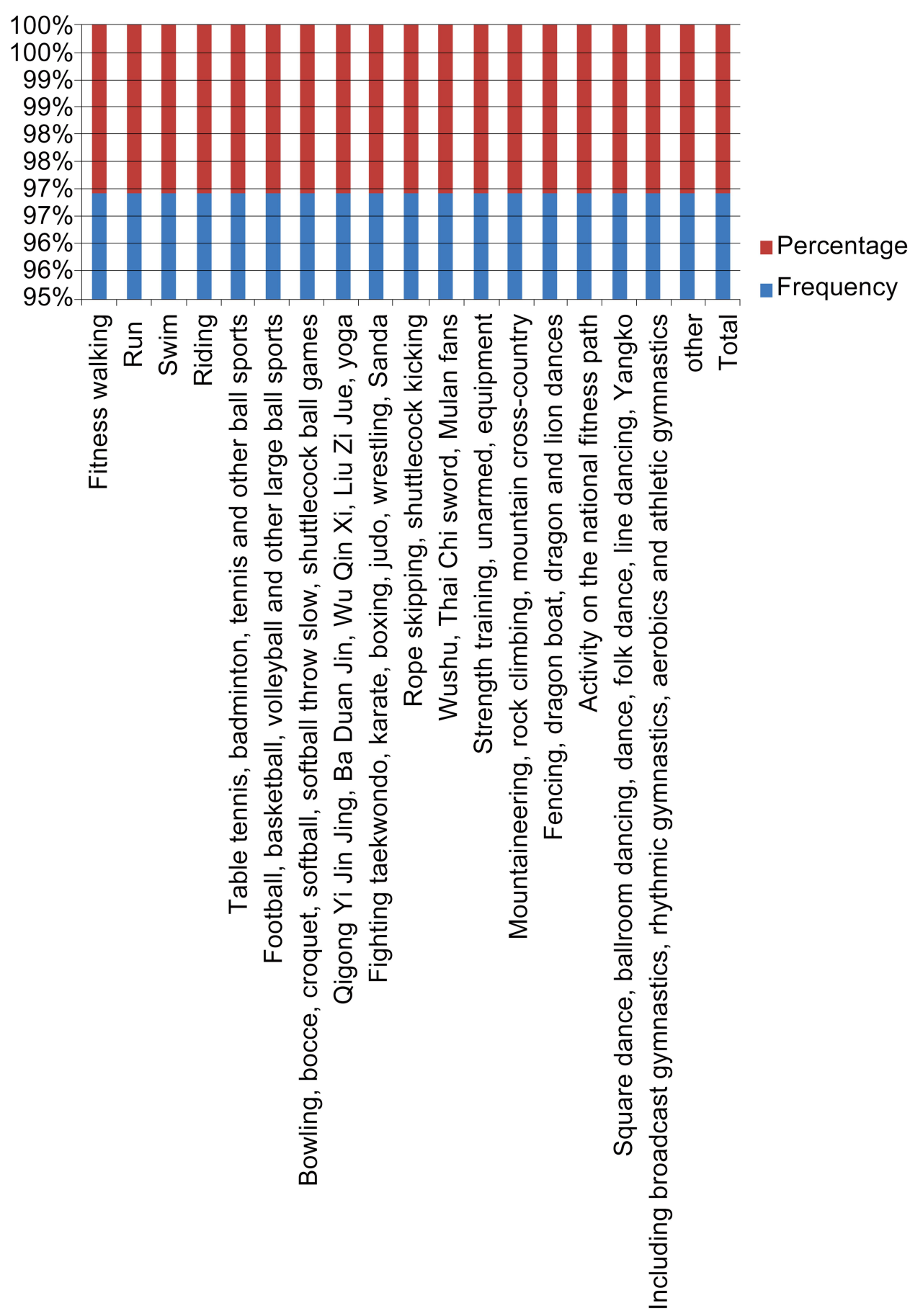

Figure 6 Types of physical activity performed by the survey participant.

popularity across the globe. Tai Ji Quan, Baduanjin, and Qigong are practiced by millions of Chinese to maintain good health. ${ }^{33}$

\section{Physical Activity Participation Form}

Figure 7 shows the survey participant's physical activity participation form. The majority of the participants, about (34.21\%), participate in their exercise, about $(32.35 \%)$ survey participants participate together with friends and colleagues, about (19.33\%) participate in the physical activity with the family. Community activity stations for physical activity are available in the Chinese society with equipment's and about (5.07\%) participate in physical activity at community physical activity stations, about $(3.60 \%)$ participants have organized exercise, about $(3.15 \%)$ participants participate in physical fitness 


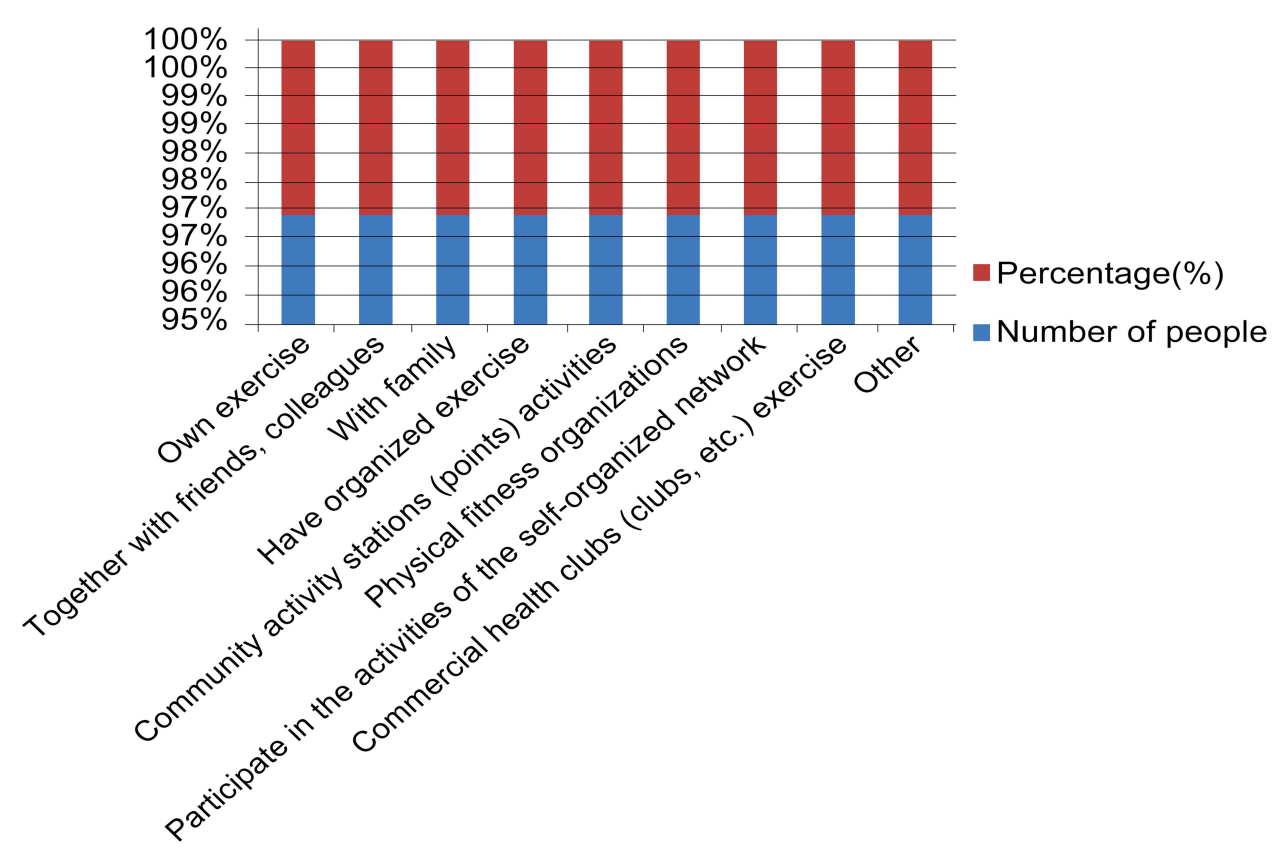

Figure 7 Physical activity participation form.

organizations (such as community health teams, sports associations, sports and fitness clubs, etc.) exercise, about (1.17\%) participants have commercial health clubs (clubs, etc.) exercise, about $(0.72 \%)$ survey participant falls in the category of other and about $(0.39 \%)$ participants participate in the activities of the self-organized network.

\section{Wilcoxon Signed-Rank Test}

A small p-value (typically $\leq 0.05$ ) indicates strong evidence against the null hypothesis so that we can reject the null hypothesis. From below Table 3, it is concluded that the null hypothesis of occupation, the primary purpose of physical exercise, physical exercise form, access to information about physical activity, types of sport for physical activity are rejected. Wilcoxon signed-rank test indicated that post-test ranks are statistically higher than pre-test ranks because the p-value is less than 0.05. Age and Education variables p-values are less than 0.05 and accepted the Alternative hypothesis. We also accepted the null hypothesis for two variables: physical activity intensity and time for physical activity because P-vales were less significant than 0.05 . Types of sports for physical activity are highly substantial with physical activity for health wellbeing.

\section{Discussion}

Physical inactivity has become a challenge due to the rising rate of non-communicable diseases. In this context, a global action plan has been launched by WHO to decrease physical inactivity and sedentary behavior in 2025 , about $10 \%$, and in 2030, about $15 \%$. Physical activity has become part and parcel of a healthy life and living. According to the WHO recommendations, an adult needs to do moderate-intensity physical activity at least 150 minutes throughout the week. Physical activity has multidimensional impacts on health, such as socio-psychological, cognitive, and prevents from non-communicable diseases. Mental acuity and depression can also be reduced through regular physical activity. Physical activity is an essential intervention in the promotion of a healthy lifestyle. Further, it is also helpful in maintaining weight (weight loss or gain weight), and it has a long-term impact on the weight regain as a preventive measure. ${ }^{34}$

\section{Physical Activity is a Medicine for Non- Communicable Diseases}

Physical activity is a natural preventive measure against different NCDs. Different NCDs rate is increasing due to physical inactivity as reported by Global Burden of Disease study that between 2005 and 2015, cancer occurrence rate increased by about $(33 \%) .{ }^{35}$ It is also expected that the cancer rate will increase by approximately $(75 \%)$ until 2030 due to demographic changes. ${ }^{36}$ The study results show that most of the participants (38.22\%) participate in fitness walking for health-being. Fitness walking 
Table 3 Wilcoxon Signed-Rank Test $(\mathrm{N}=3$ I7I)

\begin{tabular}{|l|l|l|l|l|l|}
\hline Variables & $\begin{array}{l}\text { Actual } \\
\text { Median }\end{array}$ & $\begin{array}{l}\text { Sum of Signed-Rank } \\
\text { (W) }\end{array}$ & P-value & $\begin{array}{l}\text { Summary of } \\
\text { P-value }\end{array}$ & $\begin{array}{l}\text { Significant } \\
\text { (Alpha=0.05)? }\end{array}$ \\
\hline Occupation & 240.0 & 45.00 & 0.0030 & $*$ & Yes \\
Age & 477.0 & 28.00 & 0.0156 & $*$ & Yes \\
Education & 502.0 & 21.00 & 0.0313 & $*$ & Yes \\
The primary purpose of physical exercise & 249.0 & 44.00 & 0.0040 & $* *$ & Yes \\
Physical Exercise form & 114.0 & 40.00 & 0.0031 & $* *$ & Yes \\
Access to information about physical & 139.5 & 55.00 & 0.0020 & $* *$ & Yes \\
activity & & & $<0.0001$ & $* * * *$ & Yes \\
Types of Sport for Physical Activity & 97.00 & 171.0 & 0.1250 & NS & No \\
Time for physical activity & 548.0 & 10.00 & 0.2500 & NS & No \\
Physical Activity Intensity & 946.0 & 6.000 &
\end{tabular}

Notes: $*^{*} p \leq 0.01,{ }^{*} p \leq 0.05, * * * p 0.001, * * * * p \leq 0.0001, N S p>0.05$.

has numerous health benefits and plays a vital role in the prevention of NCDs. Recent studies reported a significant relationship between walking and all causes of mortality. In this context, walking reduces about $19-30 \%$ of all factors of mortality. ${ }^{37}$ The risk of cardiovascular disease and death can be reduced in primary and secondary prevention through regular physical activity and exercise. ${ }^{38}$ CAD mortality has been found lowest, about $31 \%$ among the age group $64-84$ of men who participated in walking or cycling 20 minutes at least three times per week. ${ }^{39}$

A comparative study shows that the risk factor of stroke was reduced by about $20 \%$ to $27 \%$ through moderate or high physical activity levels among individuals. ${ }^{40}$ The survey results show that about (54.97\%) performed moderate-intensity physical activity, while about (29.83\%) performed low-intensity physical activity, and only about (15.20\%) committed high-intensity physical activity for health wellbeing. Physical activity is a catalyst for the human body, which boosts the whole body's performance, especially the immune system. About (21.51\%) survey participants participate in the physical activity to avoid different diseases. A study conducted by ${ }^{41}$ found that physical activity improves cardiovascular health through abdominal visceral fat accumulation, reduction in diastolic dysfunction, and improved endothelial vasodilator function. Physical inactivity is an accelerator of diabetes pathogenesis. Regular physical activity plays a vital role in the prevention of diabetes because it converses diabetes development process. $^{42}$

Physical inactivity is supposed a main contributory factor in obesity. According to the US health and services department, physical activity plays an important in weight loss with the low combination of calories eating food. ${ }^{43}$ The survey results are similar as mentioned above that about (20.91\%) participate in increasing physical activity, and about $(8.29 \%)$ participate in the physical activity to increase the beauty, weight loss, body sculpting. In the context of physical activity intensity, survey results indicate that about (49.79\%) performed physical activity 30 to 60 minutes, about (34.44\%) achieved 60 minutes and more for health wellbeing. According to the clinical paradigm in the context of obesity, moderate-intensity physical activity 300 minutes/week or vigorous physical activity $150 \mathrm{~min}-$ utes/week has drastic impacts on health such as to prevent obesity, weight loss, and maintenance and respiratory impairments. ${ }^{44}$ A sedentary lifestyle and imbalanced dietary food are the main factors of obesity, and both of these factors can overcome through regular physical activity.

Physical activity helps reduce depression symptoms, especially aerobic exercise, and improves the quality of life. ${ }^{45}$ In this context, survey results show that about (3.15\%) survey participants participate in physical activity for social and about (2.62\%) participants joining physical activity for recreational purposes. Both social and recreational pursuits of physical activity help in the improvement of life quality through reducing depression. Depression is a mentally disabling condition that further negatively impacts mood and is linked with diabetes, mortality, and morbidity. Depression prevalent ratio is higher in diabetic people. ${ }^{46}$ Furthermore, depression is a state of mental illness categorized as feelings of guilt, negative mood, uneasy sleep, low interest in pleasure, decline appetite, and reduced brain functioning, according to the WHO. ${ }^{47}$ Physical activity helps reduce depression symptoms, especially aerobic exercise, and improves the quality of life. ${ }^{45}$

Traditional Chinese Exercise (TCE) is a well-acknowledged concept in the Chinese society for health wellbeing. ${ }^{48}$ TCE is significantly contributing to the prevention of NCDs 
and promoting health wellbeing. ${ }^{49}$ The study results show that about $(4.79 \%)$ participate in square dance, ballroom dancing, dance, folk dance, line dancing, and Yangko (traditional Chinese exercises) for health wellbeing. About (3.34\%) participate in the rope skipping, shuttlecock kicking, about $(2.24 \%)$ participate in the broadcast gymnastics, rhythmic gymnastics, aerobics, and athletic gymnastics, about (3.06\%) participate in the Qigong Yi Jin Jing, Ba Duan Jin, $\mathrm{Wu}$ Qin Xi, Liu Zi Jue, yoga, and bowling. The study participants participated in the TCE for health wellbeing and improve the quality of life preventing from NCDs. In this context, TCE improves cardiovascular functions, psychological wellbeing, motor skills and cognitive functions. ${ }^{50}$

\section{Conclusion}

Physical activity is a medicine that improves the quality of life with the advancement of psycho-physiological functioning and cardiorespiratory fitness. Physical activity is divided into aerobic, muscle strengthening, stretching, and balancing types. Each type of physical activity positively impacts human health and plays a dynamic role in health improvement, further reducing mortality. The present study's survey results showed that Chinese people know the importance of physical activity for health and participating in moderate-intensity physical activity to improve physical fitness and prevent diseases. Most of the Chinese people perform their exercise according to their knowledge about physical activity. Physical activity is a medicine that enhances the quality of life with the advancement of psycho-physiological functioning and cardiorespiratory fitness. According to the WHO, physical activity reduces diabetes, hypertension, coronary heart disease, depression, stroke, and colon and breast cancer. The majority of the study participants performed fitness walking, which has numerous health benefits and plays a vital role in preventing NCDs. To conclude, each type of physical activity positively impacts human health and plays a dynamic role in health improvement, further reducing mortality. The current study results provide insight into understanding the increasing trend of physical activity among the Chinese population for health-being, which can also help the formation of policy regarding promoting physical activity.

\section{Ethical Approval}

The ethics committee of Soochow University, Suzhou, People's Republic of China, approved the current study's research protocol. The study participants signed consent was received, and during the informed consent procedure. Further, parental informed consent was obtained under the age of 18 . The study complied with the Declaration of Helsinki. It was also assured that all data were used only for research purposes.

\section{Funding}

Current article is a part of "Study on the Government Cultivation of Spontaneous Sports Organizations in China." Funded by the National Social Science Fund of China (16BTY033, 2016.8-2021.12, PI: DaiJianhui).

\section{Disclosure}

The author reports no potential conflicts of interest in this work.

\section{References}

1. The Public Health Agency of Sweden. The public health agency of Sweden. Folkhälsomyndigheten Myndigheten för folkhälsofrågor. Available from: http://www.folkhalsomyndigheten.se/the-publichealth-agency-of-sweden/. Accessed September 2, 2020.

2. World Health Organization Global Strategy. Available from: https:// www.who.int/dietphysicalactivity/strategy/eb11344/strategy_english_ web.pdf. Accessed May 5, 2020.

3. Bjørnarå HB, Torstveit MK, Stea TH, et al. Is there such a thing as sustainable physical activity? Scand J Med Sci Sports. 2016;27 (3):366-372. doi: $10.1111 / \mathrm{sms} .12669$.

4. Department of Health and Human Services. Physical Activity and Health: A Report of the Surgeon General. Atlanta, GA: U.S. Department of Health and Human Services, Centers for Disease Control and Prevention, National Center for Chronic Disease Prevention and Health Promotion; 1996.

5. Sadrollahi A, Hosseinian M, Alavi NM, et al. Physical activity patterns in the elderly kashan population. Iran Red Crescent Med J. 2016;18(6).

6. Warburton DE. Health benefits of physical activity: the evidence. Can Med Assoc J. 2006;174(6):801-809. doi:10.1503/cmaj.051351

7. World Health Organization. Diet and physical activity: a public health priority; 2014 Available from: https://www.who.int/dietphysi calactivity/background/en/. Accessed April 10, 2020.

8. World Health Organization. Physical activity. Available from: https:// www.who.int/health-topics/physical-activity. Accessed April 11, 2020.

9. Blair SN, Sallis RE, Hutber A, et al. Exercise therapy - the public health message. Scand J Med Sci Sports. 2012;22(4):e24-e28. doi:10.1111/j.1600-0838.2012.01462.x

10. Paterson DH, Jones GR, Rice CL. Ageing and physical activity: evidence to develop exercise recommendations for older adults. Appl Physiol Nutr Metab. 2007;32(S2E):S69-108. doi:10.1139/H07111

11. World Health Organization. NCDs global action plan on physical activity 2018-2030: more active people for a healthier world; 2019. Available from: https://www.who.int/ncds/prevention/physical-activ ity/global-action-plan-2018-2030/en/. Accessed February 20, 2020.

12. Healy GN, Wijndaele K, Dunstan DW, et al. Objectively measured sedentary time, physical activity, and metabolic risk: the Australian diabetes, obesity and lifestyle study (AusDiab). Diabetes Care. 2007;31(2):369-371. doi:10.2337/dc07-1795 
13. Malm C, Jakobsson J, Isaksson A. Physical activity and sports - real health benefits: a review with insight into the public health of Sweden. Sports. 2019;7(5):127. doi:10.3390/sports7050127

14. Lebrun C. Exercise and type 2 diabetes: American college of sports medicine and the American diabetes association: joint position statement. Yearbook Sports Med. 2011;2011:162-163. doi:10.1016/j. yspm.2011.03.038

15. Ibanez J, Izquierdo M, Arguelles I, et al. Twice-weekly progressive resistance training decreases abdominal fat and improves insulin sensitivity in older men with type 2 diabetes. Diabetes Care. 2005;28(3):662-667. doi:10.2337/diacare.28.3.662

16. Paffenbarger RS, Jung DL, Leung RW, et al. Physical activity and hypertension: an epidemiological view. Ann Med. 1991;23(3):319327. doi:10.3109/07853899109148067

17. Guthold R, Stevens GA, Riley LM, et al. Worldwide trends in insufficient physical activity from 2001 to 2016: a pooled analysis of 358 population-based surveys with 1.9 million participants. Lancet Glob Health. 2018;6(10):e1077-e1086. doi:10.1016/S2214-109X(18) 30357-7

18. Reiner M, Niermann C, Jekauc D, et al. Long-term health benefits of physical activity - a systematic review of longitudinal studies. BMC Public Health. 2013;13(1). doi:10.1186/1471-2458-13-813

19. Jr RS P, Blair SN, Lee IM. A history of physical activity, cardiovascular health and longevity: the scientific contributions of Jeremy $\mathrm{N}$ Morris, DSc, DPH, FRCP. Int J Epidemiol. 2001;30(5):1184-1192. doi:10.1093/ije/30.5.1184

20. Naci H, Ioannidis JP. Comparative effectiveness of exercise and drug interventions on mortality outcomes: met epidemiological study. BMJ. 2013;347(oct01 1):f5577.

21. Della VE, Grimaldi R, Farinaro E. L'importanza dell'attività fisica nella prevenzione delle malattie cronico degenerative [Importance of physical activity for prevention of chronic diseases]. Ann Ig. 2008;20 (5):485-493.

22. Cornelissen VA, Fagard RH. Effects of endurance training on blood pressure, blood pressure-regulating mechanisms, and cardiovascular risk factors. Hypertension. 2005;46(4):667-675. doi:10.1161/01. HYP.0000184225.05629.51

23. Kodama S, Tanaka S, Saito K, et al. Effect of aerobic exercise training on serum levels of high-density lipoprotein cholesterol: a meta-analysis. Arch Intern Med. 2007;167(10):999-1008. doi:10.1001/archinte.167.10.999

24. Colberg SR, Sigal RJ, Fernhall B, et al. Exercise and type 2 diabetes: the American college of sports medicine and the American diabetes association: joint position statement. Diabetes Care. 2010;33(12): e147-e167. doi:10.1249/mss.0b013e3181eeb61c

25. Boudou P, Sobngwi E, Mauvais-Jarvis F, et al. Absence of exerciseinduced variations in adiponectin levels despite decreased abdominal adiposity and improved insulin sensitivity in type 2 diabetic men. Eur $J$ Endocrinol. 2003;149(5):421-424. doi:10.1530/eje.0.1490421

26. Leonard WR. Size counts: evolutionary perspectives on physical activity and body size from early hominids to modern humans. $J$ Phys Act Health. 2010;7(s3):S284-S298. doi:10.1123/jpah.7.s3.s284

27. Leonard WR, Robertson ML. Nutritional requirements and human evolution: a bioenergetics model. Am J Hum Biol. 1992;4(2):179195. doi:10.1002/ajhb.1310040204

28. Babbie ER. The Practice of Social Research. Cengage Learning; 2020.

29. Godfrey AB. Juran's quality handbook; 1999. Available from: http:// dl.icdst.org/pdfs/files/7effc92a3136bc02d3041ab3399edce4.pdf. Accessed November 21, 2020.

30. Perolat J, Couso I, Loquin K, et al. Generalizing the wilcoxon ranksum test for interval data. Int J Approx Reason. 2015;56(P.A.):108121. doi:10.1016/j.ijar.2014.08.001
31. David HA, Gunnink JL. The paired $t$-test under artificial pairing. Am Stat. 1997;51(1):9-12. doi:10.1080/00031305.1997.10473578

32. Wilcoxon F. Individual comparisons by ranking. Biom J. 1945;1 (6):80-83.

33. Neufer PD, Bamman MM, Muoio DM, et al. Understanding the cellular and molecular mechanisms of physical activity-induced health benefits. Cell Metab. 2015;22(1):4-11. doi:10.1016/j. cmet.2015.05.011

34. Yach D, Hawkes C, Gould CL, et al. The global burden of chronic diseases: overcoming impediments to prevention and control. JAMA. 2004;291(21):2616-2622. doi:10.1001/jama.291.21.2616

35. Bray F, Jemal A, Grey N, et al. Global cancer transitions according to the human development index (2008-2030): a population-based study. Lancet Oncol. 2012;13(8):790-801. doi:10.1016/s1470-2045 (12)70211-5

36. Lee IM. Physical activity and cancer prevention-data from epidemiologic studies. Med Sci Sports Exerc. 2003;35(11):1823-1827. doi:10.1249/01.MSS.0000093620.27893.23

37. Lee IM, Skerrett PJ. Physical activity and all-cause mortality: what is the dose-response relation? Med Sci Sports Exerc. 2001;33(6 Suppl): S459-71. doi:10.1097/00005768-200106001-00016.

38. Giada F, Biffi A, Agostoni P, et al. Exercise prescription for the prevention and treatment of cardiovascular diseases: part I. $J$ Cardiovasc Med. 2008;9(5):529-544.

39. Stewart KJ. Exercise training and the cardiovascular consequences of type 2 diabetes and hypertension: plausible mechanisms for improving cardiovascular health. JAMA. 2002;288(13):1622-1631. doi:10.1001/jama.288.13.1622

40. Bijnen FC, Caspersen CJ, Feskens EJ, et al. Physical activity and 10year mortality from cardiovascular diseases and all causes: the Zutphen elderly study. Arch Intern Med. 1998;158(14):1499-1505.

41. Manton KG. The global impact of non-communicable diseases: estimates and projections. World Health Stat Q. 1988;41(3-4):255-266.

42. LaMonte MJ, Blair SN, Church TS. Physical activity and diabetes prevention. J Appl Physiol. 2005;99(3):1205-1213. doi:10.1152/ japplphysiol.00193.2005

43. Youssef MK. The impact of obesity on walking and physical performance. Egypt J Intern Med. 2014;26(2):40. doi:10.4103/11107782.139519

44. Zierath JR, Wallberg-Henriksson H. Looking ahead perspective: where will the future of exercise biology take us? Cell Metab. 2015;22(1):25-30. doi:10.1016/j.cmet.2015.06.015

45. Fitzmaurice C, Allen C, Barber RM, et al. Global, regional, and national cancer incidence, mortality, years of life lost, years lived with disability, and disability-adjusted life-years for 32 cancer groups, 1990 to 2015: a systematic analysis for the global burden of disease study. JAMA Oncol. 2017;3(4):pp.524-548. doi:10.1001/ jamaoncol.2016.5688

46. Wang F, Boros S. The effect of physical activity on sleep quality: a systematic review. Eur J Physiother. 2019;1-8. doi:10.1080/ 21679169.2019.1623314

47. Ploughman M. Exercise is brain food: the effects of physical activity on cognitive function. Dev Neurorehabil. 2008;11(3):pp.236-240.

48. Wang X, Pi Y, Chen B, et al. Effect of traditional Chinese exercise on the quality of life and depression for chronic diseases: a meta-analysis of randomized trials. Sci Rep. 2015;5(1):15913. doi:10.1038/ srep 15913

49. Stevens JA, Voukelatos A, Ehrenreich H. Preventing falls with Tai Ji Quan: a public health perspective. J Sport Health Sci. 2014;3(3):2126. doi:10.1016/j.jshs.2013.10.002

50. Pan L, Yan J, Guo Y, et al. Effects of Tai Chi training on exercise capacity and quality of life in patients with chronic heart failure: a meta-analysis. Eur J Heart Fail. 2013;15(15):316-323. doi:10.1093/ eurjhf/hfs 170 


\section{Publish your work in this journal}

Risk Management and Healthcare Policy is an international, peerreviewed, open access journal focusing on all aspects of public health, policy, and preventative measures to promote good health and improve morbidity and mortality in the population. The journal welcomes submitted papers covering original research, basic science, clinical \& epidemiological studies, reviews and evaluations, guidelines, expert opinion and commentary, case reports and extended reports. The manuscript management system is completely online and includes a very quick and fair peer-review system, which is all easy to use. Visit http://www.dovepress.com/testimonials.php to read real quotes from published authors. 